https://doi.org/10.5585/ExactaEP.v18n3.8612

\title{
Supply Chain Management e desempenho: proposição de um modelo conceitual
}

\author{
Supply Chain management and performance: \\ proposition of a conceptual model
}

Daniel Bruno Lopes da Silva ${ }^{1}$

Glessia Silva²

\footnotetext{
${ }^{1}$ Mestre em Engenharia de Produção pela Universidade Federal de Pernambuco - UFPE

Doutorando em Engenharia Industrial na

University of Arkansas dbruno.lopes@gmail.com
}

${ }^{2}$ Doutora em Administração de Empresas pela Fundação Getulio Vargas - FGV/EAESP Professora Adjunta da Universidade Federal de

Sergipe - UFS glessiasilva@hotmail.com

\begin{abstract}
Resumo
A gestão da cadeia de suprimentos é entendida como uma abordagem holística e integradora que considera a colaboração entre todos os membros para a coordenação do fluxo total em uma cadeia de valor. Nesse contexto, a avaliação do desempenho torna-se vital para a gestão da cadeia de suprimentos no mercado global, onde a concorrência entre empresas perde lugar para a concorrência entre cadeias de suprimentos. A avaliação do desempenho é uma ferramenta gerencial indispensável e ajuda a promover uma gestão estratégica e um controle contínuo da consecução dos objetivos. Contudo, a implementação de métricas para avaliação do desempenho é um processo complexo e os sistemas de medição do desempenho, de maneira geral, apresentam limitações importantes. Dessa forma, este ensaio teórico apresenta um modelo de medição do desempenho baseado em ferramentas para contornar as principais limitações encontradas nos modelos tradicionais de avaliação do desempenho de cadeias de suprimentos.
\end{abstract}

Palavras-chave: Cadeia de suprimentos. Sistema de medição do desempenho. Modelo de avaliação.

\begin{abstract}
Supply chain management is seen as a holistic and integrative approach that considers the colaboration among all of the supply chain members to coordinate the total flow in the supply chain. In this context, the performance evaluation becomes vital for the supply chain management on the global market, where the competition between companies is replaced by the competition between supply chains. The performance evaluation is an indispensable managerial tool and helps to promote an strategic management and a continuos control over the goals achievement. However, the implementation of metrics for performance evaluation is a complex process and the performance measurement systems generally present important limitations. Thus, this theoretical essay presents a performance measurement system based on tools to work around the main limitations found on the principal performance measurement systems.
\end{abstract}

Keywords: Supply chain. Performance measurement system. Evaluation model. 


\section{Introdução}

O campo que veio a ser conhecido como Supply Chain Management (SCM) provém da observação das vantagens obtidas com a cooperação entre elementos de cadeias de valor na década de 1990 (Beske, \& Seuring, 2014; Guersola, Lima, \& Steiner; 2018; Tan, 2001; Zhou, Chong, \& Ngai, 2015).

O conceito de SCM ganha força com a globalização dos suprimentos, que lança as companhias na busca por uma maneira mais eficiente de coordenação de suas operações, estreitando laços com fornecedores e clientes e promovendo vantagem em termos de tempo e qualidade (Guersola et al., 2018; Mentzer, DeWitt, Keebler, Min, Nix, Smith, \& Zacharia, 2001; Meredith \& Pilkington, 2018).

Supply Chain Management considera todas as organizações dentro da cadeia de valor como uma unidade virtual de negócios (Meredith \& Pilkington, 2018; Tan, 2001). Para Thomas e Griffin (1996), SCM é a gestão de materiais e fluxo de informações dentro das companhias e entre as companhias. Cooper e Ellram (1993) definem Supply Chain Management como uma filosofia integrativa de gestão do fluxo total de um canal de distribuição do fornecedor até o consumidor final. Elas localizam SCM conceitualmente entre sistemas completamente integrados verticalmente, ou seja, sistemas nos quais as funções são desempenhadas dentro de uma mesma organização, e aqueles em que cada membro opera de forma completamente independente. Neste ponto é importante observar que raramente se terá sistemas completamente integrados verticalmente. Com efeito, a orientação teórica atual aponta justamente na direção contrária, para o enxugamento das atividades corporativas, concentrando-se energia organizacional nas competências-chave e terceirizando-se atividades secundárias (Ellram \& Cooper, 2014; Kannan \& Tan, 2005; Tan, Kannan, Handfield, \& Ghosh, 1999).

Desta forma, verifica-se que, majoritariamente, empresas serão elementos de uma ou mais cadeias de suprimentos. Uma cadeia de suprimentos é entendida como um conjunto de três ou mais entidades diretamente envolvidas com o fluxo de produtos, serviços, finanças e/ou informações de uma fonte para um consumidor (Mentzer et al., 2001). Vale ressaltar, assim, que toda empresa participa de uma cadeia de suprimentos queira ela ou não (Mentzer et al., 2001).

A importância da Supply Chain Management se torna cada dia mais evidente (Krause, Luzzini, \& Lawson, 2018). Definitivamente, existe uma necessidade de integração através da cadeia de suprimentos que vai bem além da integração proposta nos termos básicos da logística (Cooper, Lambert, \& Pagh, 1997; Michalski, Montes-Botella, \& Narasimhan, 2018). Cooper e Ellram (1993) identificam três razões principais para o engajamento de empresas em Supply Chain Management: reduzir investimentos em estoque na cadeia, aumentar o serviço ao consumidor, e ajudar a desenvolver vantagem competitiva para os integrantes do canal. 
Adicionalmente, SCM tem ajudado empresas a sobreviver sob pressão constante do mercado em geral, ao passo que atingem a meta de elevar a satisfação do consumidor final por meio da melhoria contínua do desempenho em todos os pontos da cadeia (Agami, Saleh, \& Rasmy, 2012).

Neste momento, destaca-se a importância de indicadores de desempenho relacionados à SCM (Kazancoglu, Kazancoglu, \& Sagnak, 2018; Mumtaz, Ali, \& Petrillo, 2018). Essa importância é ressaltada por Croxton, Garcia-Dastugue, Lambert e Rogers (2001). Eles explicitam a necessidade do acompanhamento e mensuração do desempenho em cada um dos oito processos-chave da gestão da cadeia de suprimentos, e, assim como Kannan e Tan (2005) e Gunasekaran, Patel e McGaughey (2004), apontam, ainda para a necessidade de mais estudos em métricas de desempenho. Esses autores argumentam que muitas companhias não têm conseguido maximizar o potencial de suas cadeias de suprimentos porque têm falhado em implementar medidas e indicadores de desempenho necessários para a integração completa da cadeia e maximização da eficiência e eficácia.

A busca das organizações por capacidades e recursos da cadeia de suprimentos que tragam produtos e serviços mais rapidamente ao mercado, ao menor custo possível, com características adequadas e maior valor (Arunachalam, Kumar, \& Kawalek, 2018; Kwak, Seo, \& Mason, 2018; Tan, 2001) aumenta ainda mais a necessidade de estudos que tratem de desempenho em SCM (Migdadi, Zaid, Yousif, \& Almestarihi, 2018).

Dessa forma, este ensaio teórico objetiva propor um modelo de avaliação de desempenho em SCM a partir de uma avaliação dos principais modelos existentes e suas limitações. A complexidade das cadeias de suprimentos aumenta a dificuldade em se estabelecer modelos de desempenho adequados (Guersola et al., 2018; Kazancoglu et al., 2018). Assim, a proposta deste artigo é apresentar um modelo conceitual mais próximo das atuais necessidades das cadeias de suprimentos.

\section{Supply Chain Management e desempenho}

Organizações competitivas têm focado nas pontencialidades da cadeia de suprimentos como um todo para ganhar vantagens competitivas em termos de custo, velocidade, qualidade e valor agregado (Gunasekaran, Patel, Tirtiroglu, 2001; Li, Ragu-Nathan, Ragu-Nathan, \& Rao, 2006; Zhou et al, 2015; Guersola et al., 2018).

Nesse contexto, as medidas de performance desempenham importante papel para a consolidação e efetividade da cadeia de suprimentos (Kazancoglu et al., 2018; Migdadi et al., 2018). As companhias não podem mais buscar a otimização isolada de suas próprias operações, desconsiderando as relações com seus fornecedores e clientes, e as medidas de desempenho da SCM são os indicadores que dirão quão bem o sistema está funcionando (Kurien \& Quresh, 2011). O sistema de medidas de uma organização, por si só, afeta fortemente o comportamento de gestores e colaboradores (Kaplan \& Norton, 1992). Portanto, a medição do desempenho é uma ferramenta 
gerencial indispensável e fornece à cadeia uma gestão estratégica e um contínuo controle da consecução de seus objetivos (Agami et al., 2012).

Apesar de experimentar um grande desenvolvimento, a medição do desempenho em uma cadeia de suprimentos continua apresentando algumas questões importantes que precisam ser estudadas e entendidas para que soluções gerais sejam esboçadas (Migdadi et al., 2018; Tangen, 2004, 2005).

Uma porção considerável das companhias continua baseando-se apenas nos tradicionais indicadores puramente financeiros (Gelsomino, Mangiaracina, Perego, \& Tumino, 2016; Tangen, 2004). Entretanto, avaliações baseadas em tais critérios não conseguem representar eficientemente as mais novas gerações de SCMs. Essas complexas cadeias de colaboração buscam uma variedade de benefícios, incluindo os financeiramente intangíveis (Bhagwat \& Sharma, 2007; Kwak et al., 2018).

Além disso, Migdadi et al. (2018) e Tangen (2004) chamam a atenção para falta de estudos que atentem para os detalhes práticos das medições de desempenho na cadeia de suprimentos. Eles também argumentam que o corpo conceitual encontra-se bem fundamentado, mas o enfoque da praticidade encontra-se carente, de forma que é necessário mais trabalhos focados em traduzir os panoramas conceituais em aplicação prática para uma determinada companhia, principalmente no nível operacional.

As discussões sobre as medidas de desempenho de SCMs se expandem ainda por diferentes aspectos. Algumas das principais linhas de desenvolvimento de pesquisas relativas às medições de desempenho em SCM são a avaliação do balanço entre medidas financeiras e medidas não financeiras (Kaplan \& Norton, 1992; Tangen, 2004; Bhagwat \& Sharma, 2007); os estudos dos impactos e efeitos da SCM (Cooper \& Ellram, 1993; Li et al., 2006; Miguel \& Brito, 2011; Tan et al., 1999); e análise da integração de setores e/ou funções dentro da SCM (Prajogo \& Olhager, 2011; Kim, 2006), bem como da própria SCM com outras filosofias e práticas (Kannan \& Tan, 2005; Ellram \& Cooper, 1990).

Os estudos dos efeitos da SCM, em geral, têm realçado a importância da mensuração e acompanhamento do desempenho da cadeia de suprimentos e demonstrado, na prática, algumas das principais vantagens do engajamento na gestão da cadeia (Guersola et al., 2018).

Wisner (2003) obteve relevantes indicações quanto às relações entre gestão de fornecedores, estratégias de relação com o consumidor, estratégia de SCM e desempenho geral da firma. Utilizando modelagem de equações estruturais em uma amostra final de 350 empresas Americanas e Européias que se declararam praticantes de SCM em maior ou menor nível, este autor encontrou indícios estatísticos de que a gestão dos fornecedores e as estratégias de relação com o consumidor afetam significantemente a estratégia da SCM, que a estratégia de SCM influencia consideravelmente o desempenho geral da organização, e que a gestão de fornecedores e as estratégias de relação com o consumidor impactam uma a outra mutuamente. 
Com base nesses achados, Wisner (2003) apresenta algumas considerações importantes. Primeiramente, as organizações não deveriam ver ou avaliar suas práticas com relação a clientes e fornecedores de forma isolada. Deveriam, pelo contrário, utilizar-se de uma abordagem sistêmica e holística, uma vez que estas organizações reconhecem que as operações internas impactam diretamente a comunicação de bens e serviços ao consumidor.

Em segundo lugar, os gestores devem estar cientes que a melhoria da habilidade de coordenação e de troca de informação com fornecedores é repassada adiante na cadeia, atingindo primeiramente os próximos nós, ou seja, os clientes imediatos. Os gestores devem, também, estar cientes de que o desenvolvimento de capacidades de relacionamentos externos em uma área afetam sinergicamente outras capacidades externas. Uma terceira consideração indica que firmas que buscam desenvolver ou refinar suas capacidades de gestão da cadeia de suprimentos devem iniciar pela melhoria das relações com nós ou parceiros imediatos na cadeia. Por fim, o autor indica que estratégias de SCM com parceiros imediatos e de segundo grau influem na performance da firma direta ou indiretamente.

Kim (2006), através de uma survey com mais de 600 empresas da Koréia do Sul e do Japão e utilizando análise fatorial confirmatória (CFA), encontrou fortes traços da influência positiva das práticas de SCM na melhoria contínua do desempenho. Este autor argumenta que a integração eficiente da cadeia de suprimentos desempenha um papel crucial na melhoria contínua de pequenas firmas, enquanto que, nas grandes empresas, o fator de maior contribuição seria uma firme relação entre as práticas de SCM e a capacidade de competição da organização.

Li et al.(2006) realizaram um estudo com 196 empresas nos Estados Unidos através de questionários e de modelagem de equações estruturais, encontrando uma relação positiva entre cinco dimensões conceituais da prática de SCM, tais sejam parcerias estratégicas com fornecedores, relação com consumidor, nível de compartilhamento de informações, qualidade do compartilhamento de informações, e adiamento, e melhoria da vantagem competitiva e do desempenho organizacional.

Miguel e Brito (2011), por sua vez, confirmam a existência de uma relação positiva entre SCM e desempenho operacional em uma amostra de 103 empresas atuantes no Brasil. Através da literatura sobre prioridades competitivas, os autores conceituaram quatro dimensões para representar o desempenho operacional (custo, qualidade, flexibilidade, e entrega), e utilizando-se de modelagem de equações estruturais, encontraram uma relação positiva entre as práticas de SCM e cada uma das quatro dimensões abordadas.

Diversos outros pesquisadores expandem e aprofundam o corpo de conhecimento sobre os efeitos do gerenciamento da cadeia de suprimentos em diversos outros aspectos importantes das atividades e funções das organizações. Harland (1996) estuda a gestão da cadeia de suprimentos observando as relações, cadeias e redes; Spekman, Kamauff Jr. e Myhr (1998) investigam os efeitos da SCM a partir do ponto de vista das parcerias; Stank, Keller e Daugherty (2001) investigam os efeitos da 
colaboração dentro da cadeia de suprimentos e seus impactos no desempenho de serviços logísticos; Wong e Wong (2011) investigam como o gerenciamento do conhecimento na SCM afeta o desempenho da empresa; Boonjing, Chanvarasuth e Lertwongsatien (2015) estudam a relação entre gerenciamento de SCM e desempenho; Geng, Mansouri e Aktas (2017) estudam a relação entre gerenciamento e desempenho de SCM verdes; e Michalski et al. (2018) estudam o impacto da assimetria no desempenho em diferentes ambientes de colaboração e integração no gerenciamento e SCM.

De forma concisa, todos os trabalhos supracitados explicitam a necessidade da escolha e/ou construção e manutenção de um sistema de medições para o desempenho das atividades e práticas das empresas imersas num ambiente de SCM. Diversas outras pesquisas focam exclusivamente nos processos de escolha, construção e implantação de métricas que representem efetivamente o que a organização deseja mensurar (Akyuz \& Erkan, 2010; Beamon, 1999; Bhagwat \& Sharma, 2007; Gunasekaran et al., 2001; Gunasekaran et al., 2004; Houlihan, 1988; Kaplan \& Norton, 1992; Tangen, 2004, 2005).

Os trabalhos científicos que estudam a relação entre desempenho e SCM parecem indicar algumas tendências e alguns campos férteis para estudos futuros. Uma tendência que se consolida cada vez mais é a busca pela comprovação empírica da contribuição de sistemas de medição do desempenho na melhoria do desempenho das organizações engajadas no gerenciamento de suas cadeias de abastecimento. Diversos autores têm utilizado modelagem de equações estruturais, análise fatorial confirmatória, processo hierárquico analítico, e lógica Fuzzy entre outras técnicas, para confirmar o impacto da medição do desempenho na SCM (Kim, 2006; Kusrini, Subagyo, \& Masruroh, 2014; Li et al., 2006; Miguel \& Brito, 2011; Wisner, 2003).

Diferentemente, uma série de pesquisadores têm se concentrado em definir um modelo teórico para um sistema de medições e comprovar, na prática, a eficiência e adequação desse modelo. Dentro desse grupo, pesquisas têm se concentrado em definir quais os melhores critérios, quais os melhores indicadores, o que deve ser medido, como deve ser medido, por quem deve ser medido, em que nível deve ser medido e outras questões nessa mesma direção (Beamon, 1999; Gunasekaran et al., 2004; Kaplan \& Norton, 1992; Kusrini et al., 2014; Tangen, 2004, 2005).

De forma geral, parece haver um entendimento de que as bases teóricas do conceito de Supply Chain Management, bem como de seus conceitos internos, já se encontram fortemente solidificadas, de forma que o próximo passo lógico parece ser a comprovação prática da capacidade da SCM enquanto fonte de vantagens competitivas e meio para se alcançar a melhoria contínua efetiva. Aparentemente, há o entendimento de que o gerenciamento de cadeias de suprimentos é o fator de transformação das organizações de hoje nas organizações do futuro. Persiste ainda, entretanto, a necessidade de desenvolvimento de um modelo teórico de medições que consiga traduzir as 
estratégias da cadeia, bem como satisfazer os diversos requisitos para fornecer prontamente as informações necessárias, de forma simplificada e efetiva para a tomada de decisão.

\section{Modelos de Desempenho em SCM}

Os resultados dos processos oriundos da SCM devem ser adequadamente medidos e comparados com padrões e com metas para que os objetivos da SCM possam ser atingidos (Gunasekaran et al., 2004; Guersola et al., 2018). Frequentemente, as iniciativas de gerenciamento da cadeia de suprimentos tem falhado em razão da falta de mensuração e/ou da mensuração inadequada de seu desempenho (Arunachalam et al., 2018; Migdadi et al, 2018). Apesar do avanço no desenvolvimento de modelos de medidas de desempenho, muitas organizações continuam utilizando apenas medidas estritamente financeiras (Gelsomino et al., 2016; Tangen, 2004).

De forma geral, organizações têm falhado constantemente em maximizar os benefícios potenciais da cadeia de suprimentos, pois não têm desenvolvido métricas e medidas de desempenho adequadas que propiciem a maximização da eficiência e da eficácia da cadeia (Gelsomino et al., 2016; Gunasekaran et al., 2001; Gunasekaran et al., 2004).

Adicionalmente, as empresas necessitam dessas medidas e métricas de desempenho para testarem a viabilidade de suas estratégias, sem as quais o direcionamento para melhoria e a consecução de metas dificilmente se torna factível (Agami et al., 2012; Gunasekaran et al., 2001).

Porém, a escolha de medidas de desempenho adequadas à gestão da cadeia de suprimentos é um processo que envolve um elevado nível de dificuldade, sobretudo devido à complexidade desses sistemas de organizações (Beamon, 1999; Guersola et al., 2018; Kazancoglu et al., 2018).

Formas qualitativas de avaliação tornam-se, geralmente, vagas e difíceis de serem utilizadas num ambiente de SCM, o que faz com que medidas quantitativas sejam preferíveis. Entretanto, medidas quantitativas inadequadas tornam-se tão vagas e de pouca utilidade quanto às qualitativas mencionadas acima (Beamon, 1999; Guersola et al., 2018; Kazancoglu et al., 2018).

A dificuldade na seleção e/ou desenvolvimento de medidas adequadas não se limita à complexidade da natureza das métricas. Essa dificuldade se expande, por exemplo, pelo escopo das medidas, pois há que se considerar se devem ser mensuradas as atividades de uma única empresa ou de mais organizações e se deve ser avaliada uma única linha de produtos, ou mais (Beamon, 1999; Guersola et al., 2018; Kazancoglu et al., 2018).

Deve-se buscar, ainda, a adequação das medidas quanto ao nível, sendo necessárias medidas de desempenho estratégicas, táticas e operacionais, bem como a adequação das medidas quanto ao balanço entre critérios financeiros e não-financeiros (Gunasekaran et al., 2001). Por fim, deve-se ter em mente que a complexidade dessas medidas aumenta proporcionalmente ao número de nós ou 
níveis da cadeia e à quantidade de organizações em cada nó ou nível (Beamon, 1999; Boonjing et al., 2015).

Portanto, de acordo com Kusrini et al. (2014) pode-se classificar as várias abordagens utilizadas nos principais sistemas de medidas de desempenho de cadeias de suprimentos (Supply Chain Performance Measurement - SCPM ) em três categorias: abordagens baseadas em contexto, conteúdo e processos; abordagens baseadas na maturidade da cadeia de suprimentos; e abordagens baseadas no número de elementos medidos.

À primeira categoria se referem questões como quais são as condições de medição (contexto), o que é medido (conteúdo) e como é medido (processo). A segunda categoria pode ser dividida em três estágios, sendo o primeiro estágio relativo às primeiras fases da cadeia de abastecimento, onde o foco é a companhia e suas relações com consumidores diretos. O segundo estágio, fase intermediária da cadeia, foca em companhias que desempenham relações de cooperação com clientes e fornecedores. O terceiro estágio, cadeias de suprimentos avançadas, foca nos agentes da cadeia de suprimentos e em suas relações com outros agentes como provedores de serviços logísticos (Kusrini et al., 2014).

Na terceira categoria, as medições de desempenho são classificadas em três níveis, individual, grupal e sistêmico. No nível individual, mensura-se uma única atividade (custo, qualidade, rapidez, flexibilidade, etc.). No nível de grupo, avalia-se as atividades de um departamento, uma função ou uma unidade de negócios, e no nível de sistema mensura-se o nível de alinhamento das estratégias e da cultura corporativa (Kusrini et al., 2014).

Os sistemas de medição de desempenho da SCM podem ser classificados também entre sistemas que se baseiam apenas em custos (custos de inventário e custos operacionais, por exemplo) e sistemas que se baseiam em uma combinação entre custos e responsividade dos consumidores, o que inclui medidas como lead time, probabilidade de stockout, e taxa de preenchimento de pedidos (Beamon, 1999).

Gunasekaran et al. (2001) classificam, ainda, as medidas utilizadas em sistemas de medições em medidas estratégicas, medidas táticas e medidas operacionais, argumentando que a falta de uma distinção clara entre esses três níveis de medições é um dos principais problemas dos sistemas de medições de desempenho.

De maneira geral, a medição do desempenho em ambientes SCM têm ganhado bastante destaque no meio acadêmico e prático, sobretudo a partir dos anos 2000, partindo-se, inicialmente, de uma preocupação maior com o design e implementação de sistemas de medição do desempenho para um foco maior em como esses sistemas são usados pelas organizações (Franco \& Bourne, 2003).

Nesse sentido, Guersola et al. (2018), numa revisão de literatura recente sobre SCM e desempenho apontam que o campo de pesquisa ainda é muito imaturo e os estudos estão voltados para a medição e gestão do desempenho em SCM e em propor estruturas para resolvê-los. Akyuz e 
Erkan (2010), através de um levantamento de quarenta e dois trabalhos publicados nos principais jornais científicos, obtiveram uma indicação sólida de como as pesquisas sobre sistemas de medição do desempenho em ambientes SCM têm sido caracterizadas, classificando essas pesquisas em seis categorias temáticas: tendências e problemas gerais em cadeias de abastecimento, modelagem dinâmica, problemas de gestão do desempenho em cadeias, relação entre performance e maturidade da cadeia, priorização e dependência de KPI (Key Performance Indicators, ou indicadores-chave de performance), e os fatores humano e organizacional do desempenho de cadeias de suprimentos.

Franco e Bourne (2003), complementarmente, listam os principais fatores que possibilitam a gestão de organizações através de medições de desempenho, tais sejam: (1) cultura organizacional, (2) envolvimento e liderança da gestão, (3) sistemas de compensação; (4) educação e entendimento do sistema de medidas, (5) comunicação e reporte, (6) revisão e atualização, (7) facilidade no processamento de dados e estrutura de tecnologia da informação, (8) sistema claro de medidas, e (9) ambiente empresarial, o que inclui aspectos como posicionamento geral da empresa no mercado, foco no curto ou longo prazos e regulamentações governamentais. Esses autores, no entanto, chamam a atenção para uma falta de consenso sobre o papel e a importância das compensações atreladas às medições do desempenho, uma vez que não existe uma indicação substancial se as compensações induzem a um comportamento positivo ou negativo dos colaboradores quanto às medidas de desempenho.

No plano prático, durante a seleção ou criação de um sistema de medição do desempenho, deve-se responder questões como o que medir, como integrar múltiplas medidas individuais em um sistema de medições, com que freqüência medir, quando e como as medições devem ser reavaliadas, entre outras questões (Beamon, 1999).

Buscando responder questões como essas, alguns modelos foram desenvolvidos. Algumas análises simplificadas e compreensivas dos principais modelos podem ser encontradas em Agami et al. (2012), Akyuz e Erkan (2010), Beamon (1999), Bhagwat e Sharma (2007), Guersola et al. (2018) e Kusrini et al., (2014).

Considerando-se o impacto, a adequação e a importância na literatura, os principais modelos de mensuração do desempenho são Balanced Scorecard (BSC) - perspectiva financeira, perspectiva de processos internos, perspectiva do consumidor, e a perspectiva de aprendizado e crescimento (Kaplan \& Norton, 1992; Bhagwat \& Sharma, 2007; Kurien \& Quresh, 2011) -, The Performance Prism satisfação dos stakeholders, estratégias, processos, capacidades e contribuições dos stakeholders (Agami et al., 2012; Tangen, 2004) -, The Performance Pyramid (SMART Model) - qualidade, entrega, cycle time e desperdício (Tangen, 2004) -, Supply Chain Operations Reference (SCOR) - confiabilidade, responsividade, flexibilidade, custos, e eficiência dos ativos (Agami et al., 2012; Stephens, 2001) -, Modelo de Medori e Steeple - qualidade, custo, flexibilidade, tempo, entrega e crescimento futuro (Medori \& Steeple, 2000) -, Hierarchical-Based Measurement System - planejamento, fonte, produção 
e entrega (Gunasekaran et al., 2004) -, e o modelo de Beamon (1999) - recursos, saída e flexibilidade (Beamon, 1999).

Os modelos baseados em medidas puramente financeiras, ou Financial Performance Measurement Systems (FPMS), como o ABC (Activity Based Costing) e o EVA (Economic Value Added ) foram desconsiderados, pois, como mencionado anteriormente, há uma crescente aceitação de que sistemas baseados em medidas puramente financeiras não conseguem representar o desempenho de uma cadeia de suprimentos de forma satisfatória, apesar da incontestável importância dessas metodologias em outros tipos de diagnósticos e avaliações, como custeios e investimentos (Agami et al., 2012; Bhagwat \& Sharma, 2007; Medori \& Steeple, 2000; Tangen, 2004).

\section{Proposição de um PMS}

O desenvolvimento do modelo estruturou-se em três etapas distintas e complementares. A primeira etapa buscou fornecer o conhecimento básico acerca dos impactos e dos efeitos do desempenho em SCM. Esta etapa originou a construção teórica realizada no tópico anterior e foi conduzido a partir de uma revisão bibliográfica em livros e artigos científicos publicados nos principais jornais e períodicos nacionais e internacionais na área de Supply Chain Management.

A segunda etapa constou do estudo e da avaliação de alguns dos principais modelos de medição de desempenho comumente citados na literatura e utilizados em contextos práticos de empresas e outras organizações. Nesse ínterin, primeiramente um levantamento de PMSs existentes foi realizado em artigos científicos que focavam na comparação de PMSs ou que traziam sugestões de métricas e de modelos, tais como em Kennerley e Neely (2003),Tangen (2005), Akyuz e Erkan (2010), Kurien e Quresh (2011), Agami et al. (2012), Striteska e Spickova (2012), Kusrini, Subagyo e Masruroh (2014), Balfaqih et al.(2016), Guersola et al. (2018).

Em seguida, alguns modelos foram priorizados através de critérios como importância, alcance de publicações e diversidade de métricas, chegando-se a uma lista final de sete modelos: o Balanced Scorecard (Kaplan \& Norton,1992), o Performance Prism (Neely et al., 2001), o Performance Pyramid (Smart Model), o Scor Model, o modelo de Medori e Steeple (2000), o Hierarquical-Based Measurement System (Gunasekaran et al., 2004) e o modelo de Beamon (1999).

$\mathrm{Na}$ terceira etapa, as principais limitações desses modelos foram investigadas no intuito de encontrar ferramentas para contorná-las. As principais limitações são: (1) falta de um guia e/ou orientação prática quanto à seleção e implementação das métricas; (2) falta de um mecanismo estruturado e sistemático de atualização e adequação do PMS com o passar do tempo e com as mudanças no ambiente e na organização; (3) necessidade de maior destaque para a importância da perspectiva da flexibilidade na cadeia; e (4) falta de uma ferramenta ou mecanismo que force a cadeia de suprimentos a pensar nas tendências e ferramentas a serem incorporadas futuramente para 
garantir vantagens competitivas (Neely \& Bourne, 2000; Franco \& Bourne, 2003; Azfar, Khan, \& Gabriel, 2014; Guersola et al., 2018). Por fim, após a realização das etapas descritas acima, um novo modelo de avaliação do desempenho em SCM foi proposto.

O modelo proposto neste trabalho é estruturado a partir de cinco perspectivas ou dimensões: financeiro, flexibilidade, adequação e atualização, inovação e incorporação, e estrutura de tecnologia da informação. Uma representação desse modelo pode ser observada na Figura1.

Figura 1 - PMS proposto

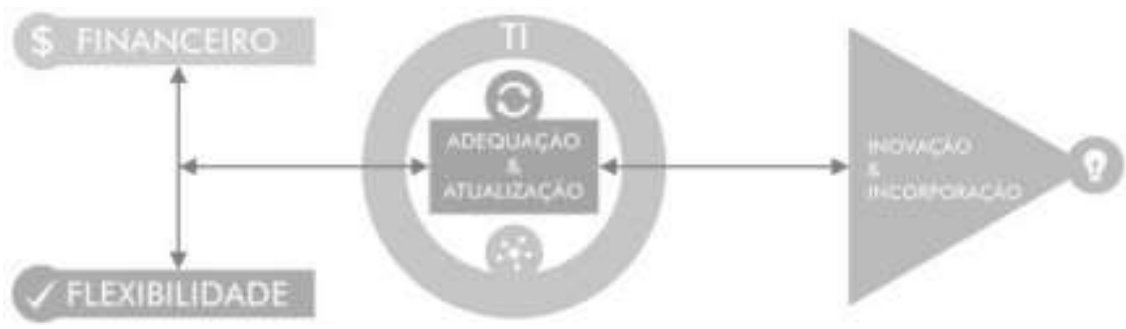

Fonte: Elaborada pelos autores

\subsection{Financeiro}

Nas últimas décadas, mais executivos têm percebido a importância estratégica da SCM e as vantagens competitivas que uma cadeia de suprimentos bem gerenciada pode trazer para a organização. Dessa forma, esses executivos precisam conhecer bem as contribuições que a SCM é capaz de trazer para o desempenho financeiro da empresa, bem como precisam saber como direcionar os investimentos na cadeia e justificar despesas relevantes (Shi \& Yu, 2013). De fato, pesquisas têm mostrado que o desempenho financeiro de uma organização pode ser consideravelmente melhorado através de uma SCM efetiva. Essa melhoria é resultado de menores custos e maior eficiência nos processos da cadeia (Greer \& Theuri, 2012).

Dessa forma, o modelo proposto tem como uma de suas perspectivas a avaliação constante do desempenho financeiro da organização. De maneira geral, a perspectiva financeira é o sistema mais antigo de avaliação do desempenho empresarial e existem diversos índices que traduzem aspectos financeiros diversos (Brigham \& Ehrhardt, 2014; Brigham \& Houston, 2015; Gitman \& Zutter, 2012)

Brigham e Houston (2015) explicam que, em geral, todos esses índices são importantes, mas, dependendo da situação particular de uma organização, alguns índices serão considerados prioritários. Por exemplo, se uma organização tomou muito capital emprestado no passado e seus débitos agora a ameaçam com uma possível falência, os índices de gestão de débitos serão prioritários. Por outro lado, se uma empresa expandiu muito rapidamente e agora apresenta excesso de estoque e capacidade produtiva superdimensionada, os índices de gestão dos ativos serão mais importantes. 
Nesse ponto, a especificidade da organização torna-se prioritária em relação à generalidade do modelo, de modo que a seleção das métricas deve ser fortemente baseada nas características e necessidades específicas da empresa. Observa-se que, de maneira geral, a perspectiva financeira já possui uma base teórica e prática sólida e robusta, com padrões globalmente aceitos (ROI, ROE, ROA, EBTIDA, etc). Adicionalmente, grande parte (se não a totalidade) das empresas utiliza métricas de avaliação financeira em maior ou menor grau, inclusive por questão fiscal.

Contudo, cabe ressaltar que também as métricas financeiras devem ser analisadas, atualizadas e adequadas conforme as mudanças na empresa e no ambiente externo. Isso significa que também a perspectiva de avaliação financeira deve ser atualizada e adequada constante e sistematicamente através da perspectiva de adequação e atualização.

Esta perspectiva engloba como métrica: índices de liquidez (indica a capacidade da organização pagar seus débitos de curto prazo); índices de atividade (indica quão eficiente uma empresa está usando seus ativos); índices de endividamento (indica como a empresa tem financiado seus ativos, bem como sua capacidade de pagar os débitos de longo prazo); índices de rentabilidade (indica quão lucrativa é a firma operando e utilizando seus ativos); e índices de valor de mercado (indica o que os investidores pensam sobre a organização e seu futuro).

\subsection{Flexibilidade}

Outra dimensão do PMS proposto é a avaliação da flexibilidade. A flexibilidade é uma dimensão essencial da gestão da cadeia de suprimentos e auxilia as organizações a acomodarem diversas externalidades, bem como as imprevisões internas.

No modelo proposto, essa avaliação é realizada através das métricas propostas por Beamon (1999) e Slack, Brandon-Jones e Johnston (2013), tais sejam flexibilidade de volume, flexibilidade de entrega, flexibilidade de mix de produtos e flexibilidade de novos produtos.

A medida de flexibilidade de volume, $F_{v}$, é proposta da seguinte forma: assume-se que a demanda $D$ é uma variável randômica com distribuição aproximadamente normal $\left(D \sim N\left(\mu_{D}, \sigma^{2}\right)\right)$ e são definidos os valores do mínimo volume rentável $\left(O_{\min }\right)$ e do máximo volume rentável $\left(O_{\max }\right)$ durante um determinado período de tempo. Considerando que a cadeia de suprimentos tenha dados suficientes sobre os volumes da demanda, os parâmetros da distribuição $D$ correspondentes à demanda média e a variância média podem ser estimados por $D$ e $S^{2}$.

A flexibilidade de entrega, por sua vez, mede a capacidade de uma cadeia de suprimentos (SC) de acomodar pedidos não planejados e pedidos especiais. Assim, define-se $t *$ como o período atual, Lj como o último período de tempo no qual a entrega do produto j pode ser realizada, e Ej o período mais antecipado durante o qual a entrega de $\mathrm{j}$ pode ser realizada. 
Flexibilidade de mix mede a variedade de bens que podem ser produzidos durante um determinado período, ou o tempo de resposta entre mudanças no mix de produtos. As métricas para ambos os casos são: $F m=N(t)$, onde $N(t)$ é o número de diferentes tipos de produtos que podem ser produzidos em um determinado período de tempo t, com $t>0$ e $N \in I+; F m=T i j$, onde Tij é o tempo de mudança necessário para passar do mix de produto i para o mix de produto j, com Tij $>0$ para qualquer ie $\mathrm{j}$.

E a flexibilidade de novos produtos pode ser entendida como a facilidade com a qual uma organização consegue introduzir novos produtos no sistema. Para a métrica baseada no tempo, temse a fórmula: $\mathrm{Fn}=\mathrm{T}$, onde $\mathrm{T}$ é o tempo necessário para a implementação do novo produto, com $T>0$. Para a métrica baseada no custo, tem-se $F n=C$, onde $C$ é o custo incorrido na introdução de um novo produto, com $\mathrm{C}>0$.

Cabe destacar, em relação a essa dimensão do PMS, que de forma intuitiva a avaliação da flexibilidade permeia diversos setores da organização e fornece uma visão balanceada da capacidade dessa organização de acomodar as incertezas. Também nesta perspectiva a necessidade de atualização e adequação se faz presente. Conforme a organização passa por mudanças de natureza interna ou externa e conforme a organização incorpora novas ferramentas e novos paradigmas, a perspectiva da avaliação da flexibilidade precisa adequar-se e atualizar-se para continuar criando valor à medida que fornece uma visão consistente da organização e auxilia nas tomadas de decisão. Além disso, cabe destacar que diferentes aspectos da flexibilidade ganham maior ou menor destaque dependendo de diversos fatores como tipo de indústria ou setor, tipo de cadeia e até da posição da empresa em relação à cadeia de suprimentos.

\subsection{Adequação e atualização}

A falta de mecanismos para atualização e adequação de um sistema de avaliação do desempenho é apontada como um dos principais motivos para as falhas de um PMS (Kennerley \& Neely, 2002, 2003; Nudurupati et al., 2011). Uma ferramenta para contornar esse problema é o framework apresentado por Kennerley e Neely (2003) e que consiste em duas fases. A primeira fase foca nos fatores ou agentes facilitadores que afetam a forma como um PMS muda ao longo do tempo e nas etapas envolvidas nessa mudança. Os agentes facilitadores da mudança são o processo, as pessoas, o sistema e a cultura e as etapas da evolução são a reflexão, a modificação e a aplicação.

Figura 2 - PMS proposto

\begin{tabular}{|c|l|c|l|l|}
\hline & Processo & Pessoas & Cultura & Sistemas \\
\hline Refletir & Em desenvolvimento & Desenvolvido & Desenvolvido & \\
\hline Modificar & Em desenvolvimento & Básico & Desenvolvido & \\
\hline Aplicar & Em desenvolvimento & Desenvolvido & Desenvolvido & Em desenvolvimento \\
\hline
\end{tabular}

Fonte: Adaptado de Kennerley e Neely $(2002,2003)$. 
A segunda fase foca na gestão da evolução do PMS e apresenta três elementos que devem ser gerenciados para garantir a evolução e adequação de um PMS: (1) medidas individuais que quantifiquem a eficiência e a efetividade das ações, (2) um conjunto de medidas combinadas que permitam acessar o desempenho geral da organização, e (3) uma estrutura de apoio que possibilite a coleta, organização, análise, interpretação e disseminação dos dados (Kennerley \& Neely, 2003).

Assim, a dimensão da adequação e atualização é incorporada ao modelo proposto e desempenha papel central, aplicando o mecanismo de atualização e adequação sistemática a todas as outras dimensões do modelo.

\subsection{Inovação e incorporação}

Uma das limitações verificadas na maioria dos PMSs analisados é a falta de uma perspectiva ou ferramenta de avaliação da inovação. Organizações necessitam cada vez mais inovar e absorver novos métodos e paradigmas para satisfazer as necessidades dos consumidores e adaptar-se à dinamicidade e agilidade do mercado global, de maneira que a capacidade de inovação de uma organização é um dos fatores mais importantes para seu desempenho (Chan, Nayak, Raj, Chong, \& Manoj, 2014; Ivanov \& Avasilcăi, 2014; Prahalad \& Mashelkar, 2010).

Nesse contexto, cabe ressaltar duas observações importantes: (1) um PMS deve ser mais que apenas informativo, ele deve ser capaz de fornecer informações que guiem ações (Neely, Gregory, \& Platts, 1995; Neely \& Bourne, 2000; Nudurupati et al., 2011), (2) a simples adoção de uma inovação não garante sua utilização e os ganhos estratégicos associados a essa inovação (Hazen, Overstreet, \& Cegielski, 2012).

Dessa forma, o modelo proposto apresenta uma perspectiva de avaliação da inovação e da incorporação efetiva de novas ferramentas. Essa avaliação é realizada através de duas ferramentas, sendo a primeira voltada para as mudanças no ambiente externo e a segunda voltada para a incorporação de inovações nos procedimentos e rotinas da organização.

A primeira ferramenta indicada é uma matriz de priorização a ser montada rotineiramente durante os planejamentos (semestrais, anuais, etc) da organização. Essa matriz confronta as novas ferramentas, métodos e paradigmas detectados no ambiente externo com critérios específicos importantes para a organização através de uma escala, de forma que a soma dos valores obtidos em cada critério permite a priorização de projetos, guiando os investimentos da organização nessa direção.

Essa ferramenta leva a organização adotante a manter, sistematicamente, um olhar para o que está acontecendo fora da organização através de benchmarking, inteligência competitiva e monitoramento ambiental. 
A segunda ferramenta avalia todo o processo de adoção de uma inovação pela organização. Proposto por Hazen et al. (2012) como forma de unificar diversos estudos sobre difusão de inovações, como os estudos de Rogers (2003), esse modelo considera que três estágios preenchem a lacuna entre a adoção de uma inovação e sua efetiva incorporação: aceitação, rotinização e assimilação. A fase de aceitação foca em como os membros percebem uma inovação na organização. Para esse estágio, Hazen et al. (2012) propõem quatro medidas ou dimensões de avaliação: expectativa de performance, expectativa de esforço, influência social, e condições facilitadoras. O estágio de rotinização avalia até que ponto o sistema de governança de uma organização está adequado para acomodar a inovação. Por fim, a assimilação, para o propósito deste modelo, é definida como o grau em que o uso da inovação se difunde através dos processos organizacionais e das atividades associadas a esses processos.

Essa perspectiva de avaliação apresenta uma relação direta e simbiótica com a perspectiva de inovação e adequação, complementando-lhe o efeito e tendo o seu próprio efeito potencializado por ela. Essa perspectiva também se relaciona diretamente com as perspectivas da flexibilidade e do desempenho financeiro.

Contudo, neste ponto, observa-se, mais uma vez, que uma estrutura adequada de tecnologia de informação e comunicação (TIC) se faz necessária, como já foi verificado em outras dimensões do modelo e de acordo com estudos como os de Kennerley e Neely (2002), Marchand e Raymond (2008), Nudurupati et al. (2011) e Chan et al. (2014).

Dessa forma, o último elemento a ser destacado no PMS proposto é justamente uma estrutura adequada de TIC. Esse elemento é discutido na próxima subseção.

\subsection{Tecnologia da informação e comunicação}

Um sistema de informação (SI) pode ser tecnicamente definido como um conjunto de componentes interrelacionados que coletam, processam, armazenam e distribuem informações para apoiar a tomada de decisão e controlar uma organização. Adicionalmente, sistemas de informação também podem auxiliar colaboradores e gerentes a analisar problemas, visualizar questões complexas e criar novos produtos (Laudon \& Laudon, 2014).

Complementarmente, um sistema de informações gerenciais (SIG) pode ser definido como a integração de quatro componentes fundamentais: (1) fornecedores de hardware e software, (2) empresas fazendo negócios e obtendo valor a partir da tecnologia, (3) gestores e empregados buscando atingir os objetivos da organização, e (4) o contexto legal, social e cultural da organização (Laudon \& Laudon, 2014). De forma prática, SIG pode ser entendido como o estudo de sistemas de informações, ou seja, o estudo de como pessoas, tecnologia, processos e dados trabalham juntos (Wallace, 2015). 
Ainda nesse sentido, tecnologia de informação e comunicação pode ser definida como uma família de tecnologias usadas para processar, armazenar e disseminar informações, facilitando o desempenho de atividades humanas relacionadas com informação (Zhang, 2011).

A forma de fazer negócios tem mudado com a introdução e a difusão das tecnologias de informação e comunicação. Virtualmente, não existe uma grande organização que não utilize algum tipo de sistema Enterprise Resource Planning (ERP) ou Electronic Data Interchange (EDI) atualmente (Donk, 2008).

De forma mais específica, um sistema de informações gerenciais desempenha um papel fundamental no fluxo de informações da rotina de uma organização. Informações (dados processados) e relatórios são passados aos níveis mais altos, enquanto que estratégias, metas e diretrizes são passadas aos níveis inferiores. Horizontalmente, informações cruciais são trocadas entre departamentos da organização e entre a organização e seus fornecedores e clientes (Nudurupati et al., 2011).

Em conjunto com o desenvolvimento das tecnologias de informação, incluindo tecnologias voltadas para a web, PMSs podem ser melhorados com novas funcionalidades que os permitam ir além de simplesmente auxiliar na medição do desempenho, promovendo um suporte mais extensivo e customizado para o processo de tomada de decisão (Marchand \& Raymond, 2008).

A perspectiva de sistemas de informações apresenta, também, um grande impacto na natureza dinâmica e evolucionária de um PMS, propiciando métodos adequados de desenvolvimento de sistemas, bem como formas de adaptação a problemas (Marchand \& Raymond, 2008).

Essa necessidade de uma estrutura adequada de tecnologia de informação e comunicação já foi apontada em outras perspectivas do modelo. A perspectiva de adequação e atualização, bem como a de inovação e incorporação de novas ferramentas, exige que exista uma estrutura adequada para o gerenciamento de informações (Kennerley \& Neely, 2003). A simples automatização do processamento de dados influencia positivamente o desempenho do PMS à medida que torna essa atividade mais rápida e padronizada (Kennerley \& Neely, 2002; Nudurupati et al.,2011).

De maneira geral, a existência de uma configuração adequada de tecnologia da informação traz benefícios como: identificação de pontos fracos da organização, promoção de um processo de tomada de decisão facilitado e proativo, promoção de melhoria contínua, aumento da transparência e visibilidade, influência no comportamento positivo dos colaboradores (Nudurupati \& Bititci, 2005).

Portanto, destaca-se o importante papel da tecnologia da informação para o funcionamento adequado de um PMS. No modelo proposto (Figura 1), a tecnologia da informação é apontada como um facilitador indispensável, não como uma dimensão de avaliação em si. Entende-se que é praticamente impossível coordenar todas as operações relativas à gestão da cadeia de suprimentos sem uma estrutura mínima de TI. 
Verifica-se, por fim, que uma quantidade cada vez maior de sistemas de informações gerenciais apresenta ferramentas voltadas para web e para o gerenciamento do relacionamento com o cliente (CRM, do inglês Costumer Relationship Management), permitindo a avaliação da percepção do cliente quanto à empresa, além de criar vantagens competitivas em termos de marketing, como fidelização do cliente, promoções, vendas e etc (Kotler \& Armstrong, 2012; Kotler \& Keller, 2016).

Entende-se, portanto, que uma estrutura de tecnologia da informação adequada é essencial para o bom funcionamento de um PMS, além contribuir diretamente para o desempenho da organização e de sua cadeia de suprimentos.

\subsection{Análise crítica do modelo de PMS proposto}

O PMS proposto se adéqua a algumas das principais recomendações encontradas na literatura: fornece uma visão geral sucinta da organização; proporciona uma visão balanceada e multidimensional do negócio; apresenta balanço e adequação em relação aos níveis hierárquicos da empresa; apresenta um detalhamento das perspectivas e sugere métricas para facilitar o entendimento; incorpora uma ferramenta para atualização e adequação sistemáticas; e mantém também um olhar nas mudanças e tendências do ambiente externo.

Esse modelo busca harmonizar perspectivas fundamentais para integração dos elementos de uma cadeia de suprimentos e permitir uma melhor gestão desta cadeia. A perspectiva financeira avaliada em cada membro permite que se tenha uma visão geral da saúde financeira da cadeia como um todo, bem como permite direcionar investimentos e esforços monetários em projetos prioritários.

Avaliando-se os diferentes aspectos da flexibilidade em cada organização, é possível obter uma visão geral de quão preparada a cadeia está para encarar as incertezas e tribulações dos mercados. Essa avaliação permite também mapear os pontos da cadeia que apresentam maior capacidade de absorver externalidades, bem como os membros mais aptos a dedicar maior investimento em P\&D e inovação de produtos e processos.

Com as ferramentas sugeridas na perspectiva de inovação e incorporação é possível manter um olhar atento para as mudanças no ambiente externo e focar nas inovações e ferramentas com maior potencial de benefícios. Esse olhar externo é fundamental para impedir que empresas se fechem em seu ambiente interno e se tornem obsoletas. Contudo, uma vez selecionada uma inovação a ser implementada, faz-se necessário um acompanhamento amplo do seu processo de adoção para garantir que esta inovação seja completamente incorporada à rotina da organização e, da fato, crie valor para a empresa. De outro modo, os investimentos nessa inovação serão inúteis gerando, além de desperdício econômico, um clima de insatisfação e falha.

As perspectivas e as métricas devem acompanhar as mudanças da organização, provendo sempre informação atualizada e adequada para guiar ações. Nesse sentido, a perspectiva de 
adequação e atualização promove uma autoavaliação sistemática e garante que o modelo esteja sempre alinhado com os objetivos estratégicos da organização e que as informações geradas possam auxiliar o processo decisório.

Todo o fluxo de dados obtidos a partir das métricas em cada perspectiva deve ser rapidamente analisado e gerar informações úteis prontamente, o que se torna impossível sem o auxílio de uma estrutura de tecnologia da informação adequada. Dessa forma, destaca-se no modelo o papel da tecnologia da informação como um agente harmonizador dos dados gerados em cada medida, auxiliando na coleta, processamento, análise, integração e direcionamento das informações.

\section{Considerações finais}

Este ensaio teórico teve como objetivo propor um modelo de avaliação do desempenho para a gestão da cadeia de suprimentos, de maneira a contornar as principais limitações encontradas em modelos tradicionais.

O PMS proposto harmoniza uma série de métricas através de cinco perspectivas básicas, fornecendo informações para auxiliar a tomada de decisão das empresas no momento em que essas informações são necessárias. Além disso, o modelo acompanha as mudanças no ambiente externo à cadeia, mantém-se alinhado aos objetivos estratégicos das empresas através de sua atualização sistemática, avalia diversos aspectos da flexibilidade, e destaca a necessidade de uma infraestrutura adequada de TI.

Acredita-se que o modelo proposto apresenta potencial para suprir as necessidades latentes dos gestores de cadeias de suprimentos, bem como auxiliar na integração dos elementos dessas cadeias, promovendo maior eficiência operacional, melhores níveis de serviço ao cliente e melhores resultados gerais. Adicionalmente, espera-se que esta pesquisa contribua com o crescente movimento de pesquisadores no caminho da consolidação de conceitos relativos à gestão da cadeia de suprimentos, de maneira geral, e aos sistemas de avaliação do desempenho em SCM.

Destaca-se, contudo, algumas limitações do modelo proposto. Primeiramente, o modelo tem caráter prescritivo, focando mais em recomendações do que em observações práticas. Em segundo lugar, o modelo não foi submetido à validação prática em algum contexto real, deixando-se essa etapa como sugestão para pesquisas futuras.

Também como sugestão para investigações futuras, indica-se o estudo de movimentos importantes que estão tomando forma e representam campo frutífero para pesquisas em SCM e desempenho. São eles: humanitarian supply chains, big data e green supply chain. 


\section{Referências}

Agami, N., Saleh, M., \& Rasmy, M. (2012). Supply chain performance measurement approaches: Review and classification. Journal of Organizational Management Studies, 1.

Akyuz, G. A, \& Erkan, T. E. (2010). Supply chain performance measurement: a literature review. International Journal of Production Research, 48(17), 5137-5155.

Arunachalam, D., Kumar, N., \& Kawalek, J. P. (2018). Understanding big data analytics capabilities in supply chain management: Unravelling the issues, challenges and implications for practice. Transportation Research Part E: Logistics and Transportation Review, 114, 416-436.

Azfar, K. R. W., Khan, N., \& Gabriel, H. F. (2014). Performance Measurement: A Conceptual Framework for Supply Chain Practices. Procedia-Social and Behavioral Sciences, 150, 803-812.

Balfaqih, H., Nopiah, Z. M., Saibani, N., \& Al-Nory, M. T. (2016). Review of supply chain performance measurement systems: 1998-2015. Computers in Industry, 82, 135-150.

Beamon, B. M. (1999). Measuring supply chain performance. International Journal of Operations \& Production Management, 19(3), 275-292.

Beske, P., \& Seuring, S. (2014). Putting sustainability into supply chain management. Supply Chain Management: An International Journal, 19(3), 322-331.

Bhagwat, R., \& Sharma, M. K. (2007). Performance measurement of supply chain management: A balanced scorecard approach. Computers \& Industrial Engineering, 53(1), 43-62.

Boonjing, V., Chanvarasuth, P., \& Lertwongsatien, C. (2015). An Impact of Supply Chain Management Components on Firm Performance. In Proceedings of the 6th International Conference on Engineering, Project and Production Management, 555-565.

Brigham, E. F., \& Ehrhardt, M. C. (2014). Financial Management: Theory and Practice. 14. ed. [S.I.]: South-Western Cengage Learning.

Brigham, E. F., \& Houston, J. F. (2015). Fundamentals of Financial Management. Concise 8. [S.I.]: South-Western Cengage Learning.

Chan, F. T., Nayak, A., Raj, R., Chong, A. Y. L., \& Manoj, T. (2014). An innovative supply chain performance measurement system incorporating research and development (R\&D) and marketing policy. Computers \& Industrial Engineering, 69, 64-70.

Cooper, M. C., \& Ellram, L. M. (1993). Characteristics of supply chain management and the implications for purchasing and logistics strategy. The International Journal of Logistics Management, 4(2), 13-24.

Cooper, M. C., Lambert, D. M., \& Pagh, J. D. (1997). Supply chain management: more than a new name for logistics. The International Journal of Logistics Management, 8(1), 1-14.

Croxton, K. L., Garcia-Dastugue, S. J., Lambert, D. M., \& Rogers, D. S. (2001). The supply chain management processes. The International Journal of Logistics Management, 12(2), 13-36.

Donk, D. P. (2008). Challenges in relating supply chain management and information and communication technology: an introduction. International Journal of Operations \& Production Management, 28(4), 308-312.

Ellram, L. M., \& Cooper, M. C. (1990). Supply chain management, partnership, and the shipper-third party relationship. The International Journal of Logistics Management, 1(2), 1-10.

Ellram, L. M., \& Cooper, M. C. (2014). Supply chain management: It's all about the journey, not the destination. Journal of Supply Chain Management, 50(1), 8-20.

Franco, M., \& Bourne, M. (2003). Factors that play a role in "managing through measures". Management Decision, 41(8), 698-710.

Geng, R., Mansouri, S. A., \& Aktas, E. (2017). The relationship between green supply chain management and performance: A meta-analysis of empirical evidences in Asian emerging economies. International Journal of Production Economics, 183, 245-258.

Gelsomino, L. M., Mangiaracina, R., Perego, A., \& Tumino, A. (2016). Supply chain finance: a literature review. International Journal of Physical Distribution \& Logistics Management, 46(4), 348-366.

Gitman, L. J., \& Zutter, C. J. (2012). Principles of Managerial Finance. 13. ed. [S.I.]: Prentice Hall.

Greer, B. M., \& Theuri, P. (2012). Linking supply chain management superiority to multifaceted firm financial performance. Journal of Supply Chain Management, 48(3), 97-106. 
Guersola, M., Lima, E. P. D., \& Steiner, M. T. A. (2018). Supply chain performance measurement: a systematic literature review. International Journal of Logistics Systems and Management, 31(1), 109-131.

Gunasekaran, A., Patel, C., \& Tirtiroglu, E. (2001). Performance measures and metrics in a supply chain environment. International Journal of Operations \& Production Management, 21(1/2), 71-87.

Gunasekaran, A., Patel, C., \& McGaughey, R. E. (2004). A framework for supply chain performance measurement. International Journal of Production Economics, 87(3), 333-347.

Harland, C. M. (1996). Supply chain management: relationships, chains and networks. British Journal of Management, $7(1)$.

Hazen, B. T., Overstreet, R. E., \& Cegielski, C. G. (2012). Supply chain innovation diffusion: going beyond adoption. The International Journal of Logistics Management, 23(1), 119-134.

Houlihan, J. B. (1988). International supply chains: a new approach. Management Decision, 26(3), 1319.

Ivanov, C. I., \& Avasilcăi, S. (2014). Performance measurement models: an analysis for measuring innovation processes performance. Procedia-Social and Behavioral Sciences, 124, 397-404.

Kannan, V. R., \& Tan, K. C. (2005). Just in time, total quality management, and supply chain management: understanding their linkages and impact on business performance. Omega, 33(2), 153-162.

Kaplan, R. S., \& Norton, D. P. (1992). The balanced scorecard - measures that drive performance. Harvard Business Review, January-February.

Kazancoglu, Y., Kazancoglu, I., \& Sagnak, M. (2018). A new holistic conceptual framework for green supply chain management performance assessment based on circular economy. Journal of Cleaner Production, 195, 1282-1299.

Kennerley, M., \& Neely, A. (2002). A framework of the factors affecting the evolution of performance measurement systems. International Journal of Operations \& Production Management, 22(11), 1222-1245.

Kennerley, M., \& Neely, A. (2003). Measuring performance in a changing business environment. International Journal of Operations \& Production Management, 23(2), 213-229.

Kim, S. W. (2006). Effects of supply chain management practices, integration and competition capability on performance. Supply Chain Management: An International Journal, 11(3), 241-248.

Kotler, P., \& Armstrong, G. (2012). Principles of Marketing. 14 ed.. ed. [S.I.]: Pearson Prentice Hall.

Kotler, P., \& Keller, K. L. (2016). Marketing Management. 15. ed. [S.I.]: Pearson Education.

Krause, D., Luzzini, D., \& Lawson, B. (2018). Building the Case for A Single Key Informant in Supply Chain Management Survey Research. Journal of Supply Chain Management, 54(1), 42-50.

Kurien, G. P., \& Qureshi, M. N. (2011). Study of performance measurement practices in supply chain management. International Journal of Business, Management and Social Sciences, 2(4), 19-34.

Kusrini, E., Subagyo, \& Masruroh, N. A. (2014). Good criteria for supply chain performance measurement. International Journal of Engineering Business Management, 6, 9.

Kwak, D. W., Seo, Y. J., \& Mason, R. (2018). Investigating the relationship between supply chain innovation, risk management capabilities and competitive advantage in global supply chains. International Journal of Operations \& Production Management, 38(1), 2-21.

Laudon, K. C., \& Laudon, J. P. (2014). Management Information Systems: managing the digital ftrm. [S.I.]: Pearson Education Limited.

Li, S., Ragu-Nathan, B., Ragu-Nathan, T. S., \& Rao, S. S. (2006). The impact of supply chain management practices on competitive advantage and organizational performance. Omega, 34(2), 107-124.

Marchand, M., \& Raymond, L. (2008). Researching performance measurement systems: An information systems perspective. International Journal of Operations \& Production Management, 28(7), 663-686.

Medori, D., \& Steeple, D. (2000). A framework for auditing and enhancing performance measurement systems. International Journal of Operations \& Production Management, 20(5), 520-533.

Mentzer, J. T., DeWitt, W., Keebler, J. S., Min, S., Nix, N. W., Smith, C. D., \& Zacharia, Z. G. (2001). Defining supply chain management. Journal of Business Logistics, 22(2), 1-25. 
Meredith, J. R., \& Pilkington, A. (2018). Assessing the exchange of knowledge between operations management and other fields: Some challenges and opportunities. Journal of Operations Management, 60, 47-53.

Michalski, M., Montes-Botella, J. L., \& Narasimhan, R. (2018). The impact of asymmetry on performance in different collaboration and integration environments in supply chain management. Supply Chain Management: An International Journal, 23(1), 33-49.

Migdadi, M. M., Zaid, M. K. S. A., Yousif, M., \& Almestarihi, R. D. (2018). An empirical examination of collaborative knowledge management practices and organisational performance: the mediating roles of supply chain integration and knowledge quality. International Journal of Business Excellence, 14(2), 180-211.

Miguel, P. L. S., \& Brito, L. A. L. (2011). Supply Chain Management measurement and its influence on Operational Performance. Journal of Operations and Supply Chain Management, 4(2), 56-70.

Mumtaz, U., Ali, Y., \& Petrillo, A. (2018). A linear regression approach to evaluate the green supply chain management impact on industrial organizational performance. Science of the Total Environment, 624, 162-169.

Neely, A., Gregory, M., \& Platts, K. (1995). Performance measurement system design: a literature review and research agenda. International Journal of Operations \& Production Management, 15(4), 80-116.

Neely, A., Bourne, M. (2000). Why measurement initiatives fail. Measuring Business Excellence, 4(4), $3-7$.

Nudurupati, S. S., \& Bititci, U. S. (2005). Implementation and impact of IT-supported performance measurement systems. Production Planning \& Control, 16(2), 152-162.

Nudurupati, S. S., Bititci, U. S., Kumar, V., \& Chan, F. T. (2011). State of the art literature review on performance measurement. Computers \& Industrial Engineering, 60(2), 279-290.

Prahalad, C. K., \& Mashelkar, R. A. (2010). Innovation's holy grail. Harvard Business Review, 88(7/8), 132-141.

Prajogo, D., \& Olhager, J. (2012). Supply chain integration and performance: The effects of long-term relationships, information technology and sharing, and logistics integration. International Journal of Production Economics, 135(1), 514-522.

Rogers, E. M. (2003). Diffusion of Innovations. Fifth. [S.I.]: The Free Press.

Shi, M., \& Yu, W. (2013). Supply chain management and financial performance: literature review and future directions. International Journal of Operations \& Production Management, 33(10), 12831317.

Slack, N., Brandon-Jones, A., \& Johnston, R. (2013). Operations Management. 7th. ed. [S.I.]: Pearson.

Spekman, R. E., Kamauff Jr, J. W., \& Myhr, N. (1998). An empirical investigation into supply chain management: a perspective on partnerships. Supply Chain Management: An International Journal, 3(2), 53-67.

Stank, T. P., Keller, S. B., \& Daugherty, P. J. (2001). Supply chain collaboration and logistical service performance. Journal of Business Logistics, 22(1), 29-48.

Stephens, S. (2001). Supply chain operations reference model version 5.0: a new tool to improve supply chain efficiency and achieve best practice. Information Systems Frontiers, 3(4), 471-476.

Striteska, M., \& Spickova, M. (2012). Review and comparison of performance measurement systems. Journal of Organizational Management Studies, 1.

Tan, K. C., Kannan, V. R., Handfield, R. B., \& Ghosh, S. (1999). Supply chain management: an empirical study of its impact on performance. International Journal of Operations \& Production Management, 19(10), 1034-1052.

Tan, K. C. (2001). A framework of supply chain management literature. European Journal of Purchasing \& Supply Management, 7(1), 39-48.

Tangen, S. (2004). Performance measurement: from philosophy to practice. International Journal of Productivity and Performance Management, 53(8), 726-737.

Tangen, S. (2005). Improving the performance of a performance measure. Measuring Business Excellence, 9(2), 4-11. 
Thomas, D. J., \& Griffin, P. M. (1996). Coordinated supply chain management. European Journal of Operational Research, 94(1), 1-15.

Wallace, P. (2015). Introduction to Information Systems. [S.I.]: Pearson Education Inc.

Wisner, J. D. (2003). A structural equation model of supply chain management strategies and firm performance. Journal of Business Logistics, 24(1), 1-26.

Wong, W. P., \& Wong, K. Y. (2011). Supply chain management, knowledge management capability, and their linkages towards firm performance. Business Process Management Journal, 17(6), 940964.

Zhang, X., Pieter van Donk, D., \& van der Vaart, T. (2011). Does ICT influence supply chain management and performance? A review of survey-based research. International Journal of Operations \& Production Management, 31(11), 1215-1247.

Zhou, L., Chong, A. Y., \& Ngai, E. W. (2015). Supply chain management in the era of the internet of things. International Journal of Production Economics, 159, 1-3.

Recebido em: 24 abr. 2018 / Aprovado em: 11 out. 2018

Para referenciar este texto

American Psychological Association (APA)

Silva, D. B. L. da., \& Silva, G. (2020). Supply Chain Management e desempenho: proposição de um modelo conceitual. Exacta, 18(3), 561582. https://doi.org/10.5585/ExactaEP.v18n3.8612. 